\title{
Simulating Study of the Effects of the Color Pump Noise on the Two-mode Laser System
}

\author{
Youlin Xiang*, Ping He, Shan Du and Zucheng Dai \\ The Department of Physics, Kunming University, Yunnan 650214, China \\ *corresponding author, e-mail: youlinxiang@163.com
}

\begin{abstract}
The color pump noise has been introduced into a two-mode laser system and its effects on the laser system in both homogeneously and inhomogeneously broadened media have been studied. The results show that the correlation time of the color pump noise can make the distribution of the laser intensity concentrate in the region where the laser intensity have larger values, enhance the mean of the laser intensity and stabilize the the system in a homogeneously broadened two-mode laser system; meanwhile, it strengthen the laser intensity of the stronger mode, recedes the laser intensity of the weaker mode and increases the competition between two mode in a inhomogeneously broadened two-mode laser system. Comparatively, it more clear that the influences of the color pump noise on the statistical properties of laser system in inhomogeneously broadened media than that in homogeneously broadened media.
\end{abstract}

Keywords: the two-mode laser system, statistical properties, mode competition, stochastic simulation

\section{Introduction}

The two-mode ring laser has attracted a great deal of attention over last decades [1-6]. Its properties such as the intensity correlations [1-3], the first-passage-time problem [4], the backscattering in a laser gyro[5] and a full account of saturation effects[6] have been studied. The reasons that the two-mode ring laser is so attractive are its widespread use [7-9] and there exists the strong competition exhibiting between two counterpropagating traveling-wave modes.

In above works, the fluctuations of the system have been assumed as the white noises with $\delta$ correlation. However, allowing for that the fluctuations of the pump of the laser system may not be sufficiently rapid to be representable by a $\delta$-correlated noise, it is more reasonable to model it using a colored noise with auto-correlation time[10]. And it is very interesting to further discuss the effects of the colored pump noise on the laser system.

In a single-mode laser system study show that the pump noise is the colored noise can better explain the experimental data, but the pump noise color effect has not been take into account in the two-mode laser systems[1-9] .

One the other hand, in a homogeneously broadened two-mode laser system, the atoms in the gain medium have basically the same the resonant frequency and linewidth, so they response to the same frequency and make identical contribution to eh laser field, in this case the coupling coefficient $\xi$ between two modes of the laser system been assumed to 2. While, in an inhomogeneously broadened two-mode laser system, the atoms in the gain medium have different resonance frequency, so the medium responses to a frequency range response as a whole of all atoms, in this case the coupling coefficient been set to 1 when the system works at the line spectral center. 
And because of the different coupling between two laser modes in inhomogeneously and homogeneously broadened two-mode laser systems, the statistical properties of the two-mode laser system have obvious difference.

In this paper, the color pump noise is introduced into the two-mode laser system; its different effects on the steady state statistical properties of the homogeneously and inhomogeneously broadened laser cubic model have been discussed.

\section{Model and Theoretical Analysis}

The dimensionless coupled complex electric fields $E_{1}(t)$ and $E_{2}(t)$ of a two-mode laser follows Langevin equations [3]:

$$
\begin{gathered}
\frac{d E_{1}}{d t}=\left(a_{1}-\left|E_{1}\right|^{2}-\xi\left|E_{2}\right|^{2}\right) E_{1}+p(t) E_{1}+q_{1}(t), \\
\frac{d E_{2}}{d t}=\left(a_{2}-\left|E_{2}\right|^{2}-\xi\left|E_{1}\right|^{2}\right) E_{2}+p(t) E_{2}+q_{2}(t),
\end{gathered}
$$

where $a_{1}$ and $a_{2}$ are the pump parameters of the two modes, and $\xi$ is the modecoupling constant which equals to 1 and 2 when the system works in the inhomogeneous and homogeneous medium, respectively. The term $p(t)=p_{R}+i p_{I}$ is the pump fluctuation, $q_{1}(t)=q_{1 R}+i q_{1 I}$ and $q_{2}(t)=q_{2 R}+i q_{2 I}$ are the independent quantum noises. These random noise terms are assumed to be zero mean and correlation

$$
\left\langle q_{k i}(t) q_{k j}\left(t^{\prime}\right)\right\rangle=\left[\delta_{i j}+\lambda\left(1-\delta_{i j}\right)\right] P \delta\left(t-t^{\prime}\right)
$$

and

$$
\left\langle p_{i}(t) p_{j}\left(t^{\prime}\right)\right\rangle=\delta_{i j} P^{\prime} \delta\left(t-t^{\prime}\right),
$$

where $i, j=I, R$ represent the real and imaginary parts and $k=1,2 . P$ and $P^{\prime}$ are the strength of the quantum noise and pump noise, respectively. $\lambda$ is the cross-correlation coefficient which measures the degree of cross-correlation between $q_{k I}(t)$ and $q_{k R}(t)$, and $|\lambda| \leq 1$. Note that we think herein that fluctuation of the complex field can be coherent and the real and imaginary parts of the quantum noise generally originate from a common bath, thus should be cross correlated [11-12]. For the sake of convenience for analysis, we employ a new function $f\left(E_{1}, E_{2}\right)$ which represents the part of the right side of the model equations except noise terms, and it is

$$
f\left(E_{1}, E_{2}\right)=\left(a_{i}-\left|E_{i}\right|^{2}-\xi\left|E_{j}\right|^{2}\right) E_{i} .
$$

where $i, j=1,2$ and $i \neq j$. So, the model equation can be rewritten as

$$
\frac{d E_{i}}{d t}=f\left(E_{1}, E_{2}\right)+p(t) E_{i}+q_{i}(t)
$$

and $i=1,2$.

By employing $E_{k}=r_{k} e^{i \varphi k} \quad(k=1,2)$, Eq. (6) can be written in a two-dimensional form:

$$
\begin{gathered}
\frac{d r_{i}}{d t}=h\left(r_{1}, r_{2}\right)+r_{i} p_{R}(t)+\varepsilon_{r i}(t), \\
\frac{d \varphi_{i}}{d t}=p_{I}(t)+\frac{1}{r_{i}} \varepsilon_{\varphi i}(t),
\end{gathered}
$$


where

$$
\begin{aligned}
& \varepsilon_{r i}(t)=q_{1 i}(t) \sin \varphi_{i}+q_{i R}(t) \cos \varphi_{i}, \\
& \varepsilon_{\varphi i}(t)=q_{i I}(t) \cos \varphi_{i}-q_{i R}(t) \sin \varphi_{i},
\end{aligned}
$$

where $h\left(r_{1}, r_{2}\right)$ is the function about $r_{1}$ and $r_{2}$.

Obviously, both $\left\langle\varepsilon_{r i}(t)\right\rangle$ and $\left\langle\varepsilon_{\varphi i}(t)\right\rangle$ are non-zero. But a sum form of one non-zero mean part and the other zero mean part for $\left\langle\varepsilon_{r i}(t)\right\rangle$ and $\left\langle\varepsilon_{\varphi i}(t)\right\rangle$ can be obtained as follows, respectively:

$$
\begin{gathered}
\varepsilon_{r i}(t)=\frac{P}{2 r_{i}}\left(1-\lambda \sin 2 \varphi_{i}\right)+\tilde{\varepsilon}_{r i}(t), \\
\varepsilon_{\varphi i}(t)=-\frac{P}{2 r_{i}} \lambda \cos 2 \varphi_{i}+\tilde{\varepsilon}_{\varphi i}(t),
\end{gathered}
$$

where

$$
\begin{gathered}
\left\langle\tilde{\varepsilon}_{r i}(t) \tilde{\varepsilon}_{r i}\left(t^{\prime}\right)\right\rangle=P\left(1+\lambda \sin 2 \varphi_{i}\right) \delta\left(t-t^{\prime}\right), \\
\left\langle\tilde{\varepsilon}_{\varphi i}(t) \tilde{\varepsilon}_{\varphi i}\left(t^{\prime}\right)\right\rangle=P\left(1-\lambda \sin 2 \varphi_{i}\right) \delta\left(t-t^{\prime}\right), \\
\left\langle\tilde{\varepsilon}_{r i}(t) \tilde{\varepsilon}_{\varphi i}\left(t^{\prime}\right)\right\rangle=P \lambda \cos 2 \varphi_{i} \delta\left(t-t^{\prime}\right),
\end{gathered}
$$

where $r_{i}$ and $\varphi_{i}$ represent the amplitude and phase of the laser field, respectively.

Inserting Eq. (12) into Eq. (8), we have

$$
\frac{d \varphi_{i}}{d t}=-\frac{P}{2 r_{i}^{2}} \lambda \cos 2 \varphi_{i}+p_{I}(t)+\frac{1}{r_{i}} \varepsilon_{\varphi i}(t) .
$$

Comparing with Eq. (8), there appears a new term $-\left(P / 2 r_{i}^{2}\right) \lambda \cos 2 \varphi_{i}$ in Eq. (16). The phase-locking condition is

$$
\left\langle\frac{d \varphi_{i}}{d t}\right\rangle=0
$$

which leads to

$$
\lambda \cos 2 \varphi_{i}=0,
$$

and the condition for the phase lock to be stable is

$$
\frac{\partial}{\partial \varphi_{i}}\left\langle\frac{d \varphi_{i}}{d t}\right\rangle-\frac{1}{2} \frac{\partial^{2}}{\partial \varphi_{i}^{2}}\left\langle\left(\frac{1}{r_{i}} \tilde{\varepsilon}_{\varphi i}(t)\right)^{2}\right\rangle<0,
$$

which leads to

$$
\lambda \sin 2 \varphi_{i}>0
$$

Both Eq. (18) and inequality (20) lead the laser phase to be locked at a series of values of $\varphi_{i}$ for the non-zero cross-correlation coefficient $\lambda$. We call them $\varphi_{\text {is }}$, and $\varphi_{i s}=(2 n+1) \pi / 4,(n= \pm 1, \pm 2, \ldots)$ and they are determined by

$$
\lambda \sin 2 \varphi_{i s}=|\lambda| \text {. }
$$


Combining Eqs. (7) and (11) with Eq. (21), we have the Langevin equation for the amplitude $r_{i}$,

$$
\frac{d r_{i}}{d t}=h\left(r_{1}, r_{2}\right)+r_{i} p_{R}(t)+\frac{P}{2 r_{i}}(1-|\lambda|)+\tilde{\varepsilon}_{r i}(t) .
$$

By using the relation $I=r^{2}$, we have obtained two Langevin equations for the laser intensity $I_{1}$ and $I_{2}$, respectively:

$$
\begin{gathered}
\frac{d I_{1}}{d t}=2\left(a_{1}-I_{1}-\xi I_{2}\right) I_{1}+2 I_{1} p_{R}(t)+P(1-|\lambda|)+2 \sqrt{I_{1}} \tilde{\varepsilon}_{r 1}(t), \\
\frac{d I_{2}}{d t}=2\left(a_{2}-I_{2}-\xi I_{1}\right) I_{2}+2 I_{2} p_{R}(t)+P(1-|\lambda|)+2 \sqrt{I_{2}} \tilde{\varepsilon}_{r 2}(t),
\end{gathered}
$$

where noise terms $\tilde{\varepsilon}_{r k}(t)$ and $p_{R}(t)$ are zero mean and their correlation are as follows:

$$
\begin{gathered}
\left\langle\widetilde{\varepsilon}_{r k}(t) \tilde{\varepsilon}_{r k}\left(t^{\prime}\right)\right\rangle=P(1+|\lambda|) \delta\left(t-t^{\prime}\right), \\
\left\langle p_{R}(t) p_{R}\left(t^{\prime}\right)\right\rangle=P^{\prime} \delta\left(t-t^{\prime}\right),
\end{gathered}
$$

and $k=1,2$.

Obviously, $\tilde{\varepsilon}_{r k}(t)$ and $p_{R}(t)$ are white noises with $\delta$ correlation. But allowing for that the fluctuations of the pump of the laser system may not be sufficiently rapid to be representable by a $\delta$-correlated noise, it is reasonable to model it using a colored noise with an auto-correlation time[10]. Now, we assume that the colored noise representing the pump fluctuation is zero mean and exponential correlation as follows:

$$
\begin{aligned}
\left\langle p_{R}(t)\right\rangle & =0, \\
\left\langle p_{R}(t) p_{R}\left(t^{\prime}\right)\right\rangle & =\frac{P^{\prime}}{\tau} e^{-\frac{\left|t-t^{\prime}\right|}{\tau}},
\end{aligned}
$$

where $\tau$ is the auto-correlation time of the pump noise.

Here has a drawback to using colored pump noise, that is the system changes to be nonmarkov process and so has no exactly analytic results. In present article, we employ the computer simulation method to study such properties of the system as the mean, the auto-correlation function and the cross-correlation function of the laser intensity.

The expectation values of the product of the $n$th power of intensity $I_{1}$ and the $m$ th power of intensity $I_{2}$ are given by[7]

$$
\left\langle I_{1}^{n} I_{2}^{m}\right\rangle=\iint_{0}^{\infty} I_{1}^{n} I_{2}^{m} Q_{s}\left(I_{1}, I_{2}\right) d I_{1} d I_{2} .
$$

Then the mean laser intensity of two mode of the laser system are

$$
\left\langle I_{1}\right\rangle=\left\langle I_{2}\right\rangle=\iint_{0}^{\infty} I_{1} Q_{s}\left(I_{1}, I_{2}\right) d I_{1} d I_{2} .
$$

So the normalized auto-correlation function of the two-mode laser intensity are defined as

$$
\lambda_{11}(0)=\lambda_{22}(0)=\frac{\left.<\left(\Delta I_{1}\right)^{2}\right\rangle}{\left\langle I_{1}\right\rangle^{2}}=\frac{\left.<I_{1}^{2}\right\rangle}{\left.<I_{1}\right\rangle^{2}}-1 .
$$

And the normalized cross-correlation function of the two-mode laser intensity are defined as 


$$
\lambda_{12}(0)=\lambda_{21}(0)=\frac{<\Delta I_{1} \Delta I_{2}>}{<I_{1}><I_{2}>}=\frac{<I_{1} I_{2}>}{<I_{1}><I_{2}>}-1,
$$

where $\Delta I_{i} \quad(i=1,2)$ is the laser intensity fluctuation relative to the mean laser intensity and it reads

$$
\Delta I_{i}=I_{i}-<I_{i}>
$$

The computer simulation is effective to study our problem in this letter [13] and we have mastered it through the experiences in previous works. The algorithm for computer simulation developed by Fox etc.[14] is used. The details of the simulation procedure are as follows. The starting point was $I_{1}=I_{2}=0$ and the time step was 0.001 . Five thousand independent realizations were obtained, and a realization was comprised of 100000 different time steps. The simulation results show next parts of the article.

\section{Simulation Results and Statistics Analysis}

\subsection{The Probability Distribution of the Laser Intensity}
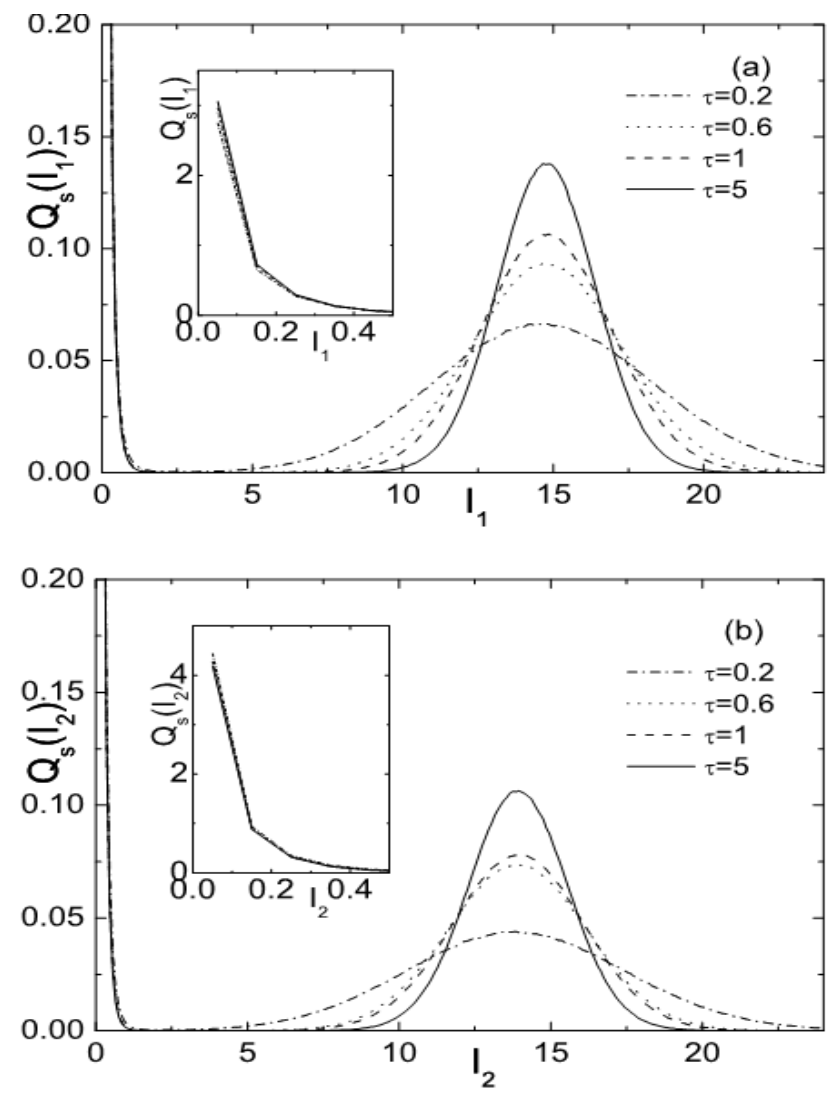

Figure 1. The stationary probability distribution of the laser intensity as a function of the laser intensity for several different values of the autocorrelation time of the colored pump noise $\tau$ in a homogeneous medium, and related parameters values: $a_{1}=15, \Delta a=0.8, \lambda=0, \xi=2, P=2, P^{\prime}=5$, (a) intensity probability distribution for mode 2. 
Figure 1 shows that The stationary probability distribution of the laser intensity as a function of the laser intensity for several different values of the auto-correlation time of the colored pump noise $\tau$ in a homogeneous medium. It's can be clearly seen that:

For laser mode 1, the laser intensity distribution in homogeneous broadening laser system has two possible areas, one area is that near the laser intensity is zero, another area is around the region which is close to the laser intensity $I_{1}=a_{1}$, which makes the laser intensity distribution may migrate between two areas. Meanwhile, the probability which the laser intensity occur in the region nearby $I_{1}=a_{1}$ gets bigger as the auto-correlation time $\tau$ increases. For laser mode 2, the similar things to what the mode 1 happens, the difference is that one of the probability of the laser intensity concentrating areas is not nearby $I_{1}=a_{1}$ but close to $I_{1}=a_{1}-\Delta a$.

\subsection{The Mean, Auto-correlation and Cross-correlation Functions of the Laser Intensity}

Figures 2-6 display the mean laser intensity, the auto-correlation and cross-correlation functions of the laser intensity of the laser systems in the homogeneous $(\xi=2)$ and inhomogeneous $(\xi=1)$ medium.

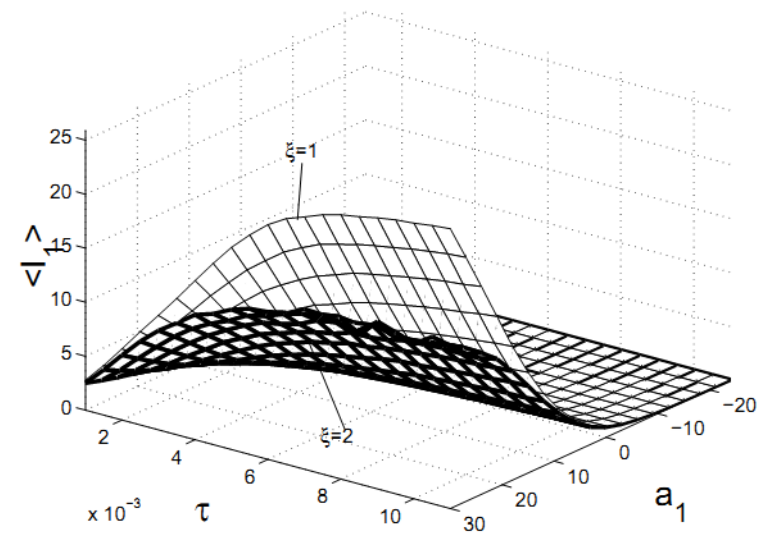

Figure 2. The mean laser intensity of the mode 1 in different medium (homogeneous and inhomogeneous medium), the other parameter

values: $\Delta a=0.8, \lambda=0, P=2, P^{\prime}=5$.

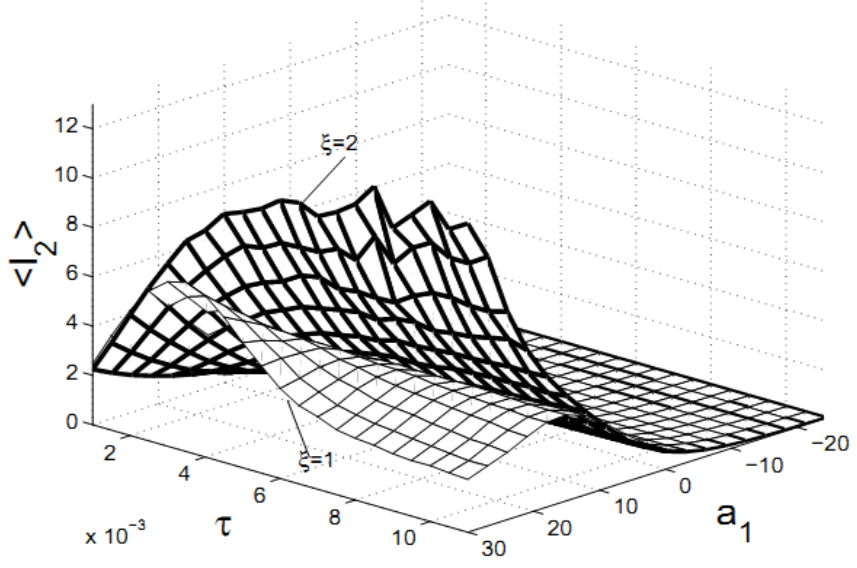




\section{Figure 3. The mean laser intensity of the mode 2 in different medium (homogeneous and inhomogeneous medium), the other parameter \\ values: $\Delta a=0.8, \lambda=0, P=2, P^{\prime}=5$.}

In Figure 2 and Figure 3, the dark grids represents the mean laser intensity of the homogeneous laser system $(\xi=2)$ and the thin grids represents the mean laser intensity of the inhomogeneous laser system $(\xi=1)$, respectively. From two figures, we can see that below and at the threshold value of the laser system, the mean light intensities for both two laser systems are not different, but above the threshold value, the changes of the mean light intensities of the laser systems work in the different medium are distinctly different.

Figure 2 gives that above the threshold value, the mean light intensities of the laser mode 1 of the inhomogeneous laser system increase faster than that of the homogeneous laser system when the auto-correlation time of the colored pump noise $\tau$ increases with certain limits, and it is more obvious when the pump parameters $a_{1}$ is increasing.

The different changes of two mean light intensities of the mode 2 in different medium show in Figure 3. This figure displays that that above the threshold value, the mean light intensities of the laser mode 2 of the homogeneous laser system increase faster than that of the inhomogeneous laser system when the auto-correlation time of the colored pump noise $\tau$ increases with certain limits, and it is indeed more obvious when the pump parameters $a_{1}$ is increasing just as that show in Figure 2. In addition, the mean light intensities of the laser mode 2 of the inhomogeneous laser system firstly increase and then decreases as increasing $\tau$ with certain limits.

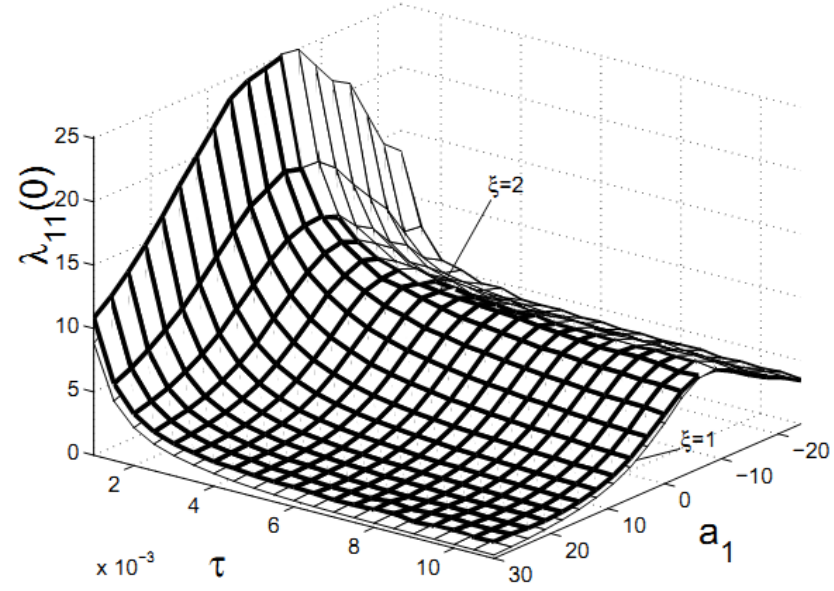

Figure 4. The auto-correlation functions of the light intensities of the mode 1 in different medium (homogeneous and inhomogeneous medium), the other parameter values: $\Delta a=0.8, \lambda=0, P=2, P^{\prime}=5$. 


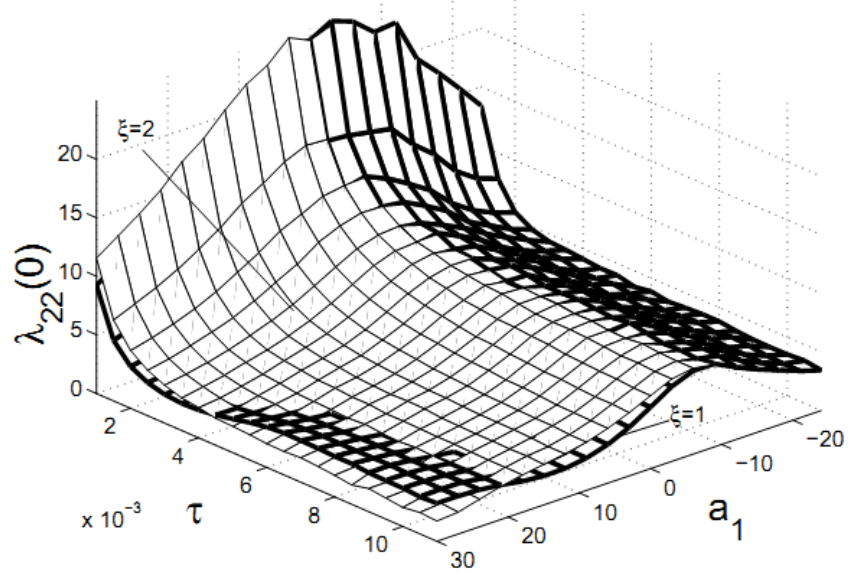

Figure 5. The auto-correlation function of the laser intensity of the mode 2 in different medium (homogeneous and inhomogeneous medium), the other parameter values: $\Delta a=0.8, \lambda=0, P=2, P^{\prime}=5$.

Figures 4 and 5 show the auto-correlation functions of the light intensities and Figure 6 displays the cross-correlation functions of the light intensities when the laser system work in homogeneous and inhomogeneous medium. Note that in Figure 4 the dark and thin grids keep the same sense as that of in Figures 2 and 3, but in Figures 5 and 6, In order to more clear exhibition, the dark grids represents the mean laser intensity of the inhomogeneous laser system $(\xi=1)$ and the thin grids represents the mean laser intensity of the homogeneous laser system $(\xi=2)$, respectively.

Figures 4 and 5 show that Below and at the threshold value of the laser system, the auto-correlation of the light intensities of the different medium are not different, but when the laster system works above the threshold value, the values of the auto-correlation function of the light intensities of the laser mole 1 in the inhomogeneous medium are smaller than that in the homogeneous medium; meanwhile, the values of the autocorrelation function of the light intensities of the laser mole 2 in the homogeneous medium gets litter and litter when the values of $\tau$ or $a_{1}$ gets bigger.

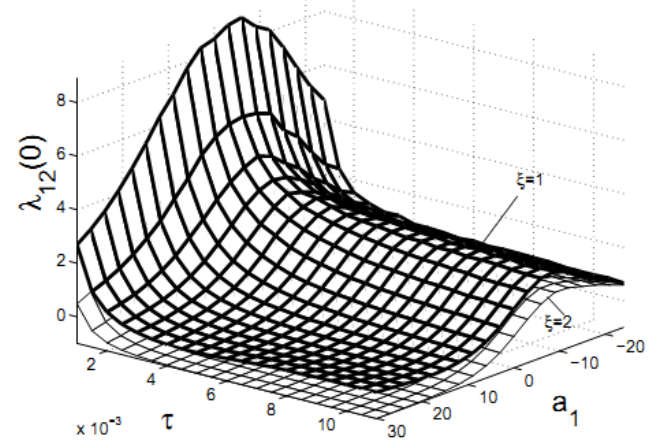

Figure 6. The cross-correlation function of the laser intensity of the mode 1 and 2 in different medium (homogeneous and inhomogeneous medium), the other parameter values: $\Delta a=0.8, \lambda=0, P=2, P^{\prime}=5$.

As for the cross-correlation function of the light intensities of two laser modes, from Figure 6, we can see it decreases as increasing values of $\tau$ or $a_{1}$ gets bigger when the 
laser systems work above the threshold value of the system, it has the maximum value near the threshold value and then gets smaller when the pump parameter decreases.

\section{Conclusion}

Then studies show that the stationary probability of the laser intensity mainly occur in two regions, the correlation time of the color pump noise $\tau$ may make the distribution of the laser intensity concentrate in the region where close to the laser intensity $I_{1}=a_{1}$ and $I_{1}=a_{1}-\Delta a$ and the values of the light intensities are larger, thus enhance the mean of the laser intensity and stabilize the the system in the homogeneously broadened twomode laser system; at the same time, the mean laser intensity of the laser mode 1 decreases and he mean laser intensity of the laser mode 2 increases when $\tau$ gets bigger, so the correlation time of the color pump noise $\tau$ may weaken the competition between the two laser modes.

\section{Acknowledgements}

This work supported by the Program for Innovative Research Team (in Science and Technology ) in University of Yunnan Province, the National Natural Science Foundation of China (Grant No.11047192), the Foundation of Yunnan Provincial Science and Technology Department of China(Grant No.2010ZC168), and the Foundation of Kunming University (Grant No.YJL11009).

\section{References}

[1] M. M. Tehrani and L. Mandel, "Coherence theory of the ring laser", Phys. Rev. A., vol. 17, no. 2, (1978), pp. 677-693.

[2] M. M. Tehrani and L. Mandel, "Intensity fluctuations in a two-mode ring laser", Phys. Rev. A., vol. 17, no. 2, (1978), pp. 694-700.

[3] S. Zhu, "Steady-state analysis of a two-mode laser with multiplicative white noise", Phys. Rev. A., vol. 45, no. 11, (1992), pp. 8148-8153.

[4] R. Roy, R. Short, J. Durnin and L. Mandel, "First-Passage-Time Distributions under the Influence of Quantum Fluctuations in a Laser", Phys. Rev. Lett., vol. 45, no. 18, (1980), pp. 486-1490.

[5] W. R. Christian and L. Mandel, "Frequency dependence of a ring laser with backscattering", Phys. Rev. A., vol. 34, no. 5, (1986), pp. 3932-3932.

[6] S. Zhu, "Saturation effects in a two-mode ring laser", Phys. Rev. A., vol, 50, no. 2, (1994), pp. 1710-1715.

[7] S. Zhu, "Steady-state analysis of a single-mode laser with correlations between additive and multiplicative noise", Phys. Rev. A., vol. 47, no. 3, (1993), pp. 2405-2408.

[8] Q. Long, L. Cao, D. J. Wu and Z. G. Li, "Phase lock and stationary fluctuations induced by correlation between additive and multiplicative noise terms in a single-mode laser", Phys. Lett. A., vol. 231, (1997), pp. 339-343.

[9] S. Z. Ke, L. Cao, D. J. Wu and K. L. Yao, "Stationary properties in a single-mode laser with cross-correlation between quantum noise terms", Phys. Lett. A., vol. 281, (2001), pp. 113-118.

[10] R. Short, L. Mandel and R. Roy, "Correlation Functions of a Dye Laser: Comparison between Theory and Experiment”, Phys. Rev. Lett., vol. 49, no. 9, (1982), pp. 647-650.

[11]A. Fulinski and T. Telejko, "On the effect of interference of additive and multiplicative noises”, Phys. Lett. A., vol. 152, no. 1-2, (1991), pp. 11-14.

[12]X. Zhou, W. Gao and S. Zhu, "Saturation effects in a two-mode ring laser with both additive and multiplicative noise", Phys. Lett. A., vol. 213, no. 1-2, (1996), pp. 43-48.

[13] I. T. Dimov, "Monte Carlo Methods for Applied Scientists, 1st Ed.", Singapore: World Scientific Press, (2008), pp. 11-14.

[14]R. F. Fox, I. R. Gatland, R. Roy and G. Vemuri, "Fast, accurate algorithm for numerical simulation of exponentially correlated colored noise", Phys. Rev. A., vol. 38, no. 11, (1988), pp. 5938-5940. 
International Journal of Signal Processing, Image Processing and Pattern Recognition Vol.8, No.8 (2015) 